\title{
BMJ Open Decision support intervention (Plan Well Guide) for patients and their substitute decision-makers to improve engagement in advance care planning: protocol for a randomised trial
}

\author{
Michelle Howard, ${ }^{\oplus 1}$ Marissa Slaven, ${ }^{1,2}$ Carrie Bernard ${ }^{3}$ Sayem Borhan, ${ }^{1}$ \\ Dawn Elston, ${ }^{1}$ Neha Arora, ${ }^{1}$ Amy Tan, ${ }^{4}$ Daren K Heyland ${ }^{5,6}$
}

To cite: Howard M, Slaven M, Bernard C, et al. Decision support intervention (Plan Well Guide) for patients and their substitute decision-makers to improve engagement in advance care planning: protocol for a randomised trial. BMJ Open 2019;9:e027897. doi:10.1136/ bmjopen-2018-027897

- Prepublication history for this paper is available online To view these files, please visit the journal online (http://dx.doi org/10.1136/bmjopen-2018027897).

Received 13 November 2018 Revised 27 August 2019 Accepted 30 August 2019
Check for updates

(c) Author(s) (or their employer(s)) 2019. Re-use permitted under CC BY-NC. No commercial re-use. See rights and permissions. Published by BMJ.

For numbered affiliations see end of article.

Correspondence to Dr Michelle Howard mhoward@mcmaster.ca

\section{ABSTRACT}

Objective The purpose of this randomised trial is to evaluate the efficacy of a decision support intervention for serious illness decisions (the Plan Well Guide decision aid; www.PlanWellguide.com) in increasing the engagement of substitute decision-makers (SDMs) in the patient's advance care planning process (ie, 'ACP engagement'), specifically the SDM's confidence and readiness for the role in the future.

Methods and analysis This study is a parallel group randomised controlled trial. We aim to enrol 90 participant dyads: patients aged 65 years and older attending outpatient healthcare settings and with indicators they would be at high risk of needing future medical decisions and their SDM. The intervention is the Plan Well Guide decision aid, administered to the patient by a facilitator, with the SDM present. The primary endpoint is change after 3 months in the SDM's ACP engagement using a validated measure of behavioural change (SDM's knowledge, contemplation, self-efficacy and readiness) to enact the role. The secondary endpoints are (1) ACP engagement of the patient measured by a validated survey; (2) change in SDM decisional conflict regarding involvement in future decision-making and (3) postintervention patient decisional conflict regarding preference for life-sustaining treatments. Primary and secondary continuous outcomes will be analysed using the linear regression. The mean difference and $95 \%$ Cls will be reported.

Ethics and dissemination Approval was received August 2017 (2017-3714-GRA) from the Hamilton Integrated Research Ethics Board. We plan to disseminate trial results in peer-reviewed journals, at national and international conferences, and via our web-based knowledge translation platforms.

Trial registration number NCT03239639; Pre-results.

\section{BACKGROUND RATIONALE}

During serious illness or at the end of life, there is often a need for decisions to be made about the use of various medical treatments, such as whether to accept life-sustaining

\section{Strengths and limitations of this study}

- This decision aid, Plan Well Guide, was developed using the International Patient Decision Aids Standards.

- The Plan Well Guide decision support intervention was developed with extensive input from patients and clinicians.

- The study evaluates the impact of the decision aid on substitute decision-makers as the primary outcome because of the likelihood that they will need to be involved in healthcare decisions for a loved one in the future.

- The study evaluates a short-term surrogate endpoint, further research on long-term outcomes would be warranted if this trial is positive.

treatments. A sizeable proportion of seriously ill older adults will experience a time when they lack the capacity to make their own medical treatment decisions, thus shifting the decision responsibility to a surrogate or 'substitute' decision-maker $(\mathrm{SDM}) .{ }^{1}{ }^{2}$ The ability of SDMs to make decisions consistent with their loved one's wishes is generally poor and needs improvement. ${ }^{3-5}$ In order to ensure the patient's prior expressed values and wishes are followed, patients and their SDM would ideally have engaged in a process to increase their 'decisional readiness'.

Advance care planning (ACP) is a process that supports adults at any age or stage of health in understanding and sharing their personal values, life goals and preferences regarding future medical care. The goal of ACP is to help ensure that people receive medical care that is consistent with their values, goals and preferences during serious and chronic illness. ${ }^{6}$ In prospective and randomised trials, ACP significantly increases 
the likelihood that clinicians and SDMs understand and apply a patient's previously expressed wishes when making medical treatment decisions, ${ }^{7-10}$ reduces hospitalisation at the end of life, results in less intensive treatments at the end of life (according to patients' wishes) and increases the use of hospice services. ${ }^{1112}$

Despite knowledge of the benefits of ACP, studies continue to document deficiencies in the quality or quantity of communication and decision-making during serious illness. ${ }^{13-17}$ Patients are seemingly able to express a preference related to the use of life-sustaining treatments, however, they report considerable decisional conflict about the preference, and there is a lack of alignment between stated values and preferences. ${ }^{18} 19$ In our survey of mostly well older adults in primary care, approximately one-third indicated that they would want only comfort care and not life-sustaining treatment if they became seriously ill and only $8 \%$ indicated a preference for cardiopulmonary resuscitation (CPR) ${ }^{19}$ suggesting that preferences may not be informed by the outcomes of various treatments for different populations. Other studies have documented the lay public's significant overestimation of the probability of survival after $\mathrm{CPR}^{20}$ and a lack of understanding of palliative care. ${ }^{21}$ These knowledge gaps may partly contribute to the creation of advance directives that do not reflect patients' values and informed preferences and are therefore unhelpful to guide clinical decision-making ${ }^{22}{ }^{23}$ or the SDM in his or her role. ${ }^{24}$

Decision aids are tools developed and promoted to increase high-quality decisions in healthcare by clearly communicating treatment options, and the benefits, harms and outcomes associated with each option, in a manner that is easily understood by lay persons. Many decision aids include a component of values clarification to help the patient construct preferences about the outcomes of treatment options in relation to their own values. In randomised studies, decision aids have generally been found to be helpful to increase patients' knowledge and the congruence between the decision and the patient's values, for a range of screening and treatment decisions. ${ }^{25}$ A systematic review of decision aids to help older patients who were facing serious illness found shortcomings. Of the 17 studies reviewed, five included a values clarification process, only one of which made trade-offs explicit, and none explicated the general difference between the intent and approaches of resuscitative/ intensive care vs medical care versus comfort care. ${ }^{26}$

SDMs can experience prolonged emotional difficulty after making decisions for a loved one when they are unsure if they have followed the loved one's wishes. ${ }^{27}$ Both patients and clinicians have identified the need to prepare SDMs as a key focus in ACP. ${ }^{28-30} \mathrm{ACP}$ tools should ideally help to prepare the SDM as well as the patient, ${ }^{31}$ and these approaches should be evaluated for their effectiveness to prepare SDM. The purpose of this randomised trial is to evaluate the efficacy of a decision aid (the Plan Well Guide tool; www.planwellguide.com) administered with a patient in the presence of the patient's SDM, in increasing the SDM's engagement in ACP, defined here as knowledge, contemplation, self-efficacy and readiness for enacting their role in the future.

\section{RESEARCH AIMS AND STUDY HYPOTHESES}

The primary objective of this study is to determine the efficacy of the Plan Well Guide decision support intervention to increase the SDM's engagement in ACP with respect to their loved one (the patient) after being present while the patient uses the decision aid. The secondary objectives are to determine the efficacy of the Plan Well Guide decision support intervention to increase ACP engagement of the patient and to describe patients' decisional conflict associated with his or her stated current preference for life-sustaining treatments after using the Plan Well Guide.

The primary outcome will be evaluated in the SDM who accompanies the patient. A separate randomised trial of the patient's decisional outcomes is reported elsewhere (unpublished) (registration at clinicaltrials. gov; NCT03434626). We will use a validated 'SDM ACP Engagement Survey' measure that asks about SDM engagement related to that role for a loved one. ${ }^{32}$ Our primary hypothesis is that the SDM's presence while the patient is guided through the Plan Well Guide will result in increased engagement in behavioural change processes concerning ACP in relation to being involved in the loved one's future decision-making, as measured in the SDM (see the Outcomes and Measures section), compared with usual care. Our secondary hypotheses are that compared with usual care, the intervention will result in increased ACP engagement in the patient, and improved decisional self-efficacy of the SDM, compared with usual care.

\section{METHODS AND ANALYSIS}

The methods are described according to the Standard Protocol Items: Recommendations for Interventional Trials reporting guidelines. ${ }^{33}$ This is a parallel group randomised controlled superiority trial with a delayed intervention control group in a 1:1 allocation ratio. Outcomes are measured at baseline before randomisation and at 12 weeks postrandomisation. Participants in the delayed intervention arm will be offered the intervention at the end of their follow-up period.

Participants are dyads consisting of a patient and the patient's identified SDM. Eligible consenting patients and SDM complete a baseline questionnaire by interview with a research assistant and are then randomised together to the immediate Plan Well Guide decision support intervention arm or the delayed intervention arm (table 1). It is possible that both people in the dyad will be eligible as patients may be each other's SDM. If both wish to participate as the SDM (ie, one dyad will count as two study participants), they will be randomised to the same group. At baseline, patients in both groups will receive a booklet 
Table 1 Randomised trial design flow chart

Eligible patients referred to research team from participating clinics:

- Age 65 years or older

- Have a health condition that would make advance care planning (ACP) relevant

- Perceived to have adequate cognitive ability to participate

- Able to communicate in English

\section{Participant screening:}

- Confirm eligibility

- Obtain name of substitute decision-maker (SDM) and permission to contact

- Verbal consent obtained of patient and SDM

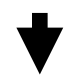

Baseline surveys administered:

- Patient and SDM Demographic Surveys

- Patient Clinical Frailty Score

- Patient ACP Engagement Survey

- SDM ACP Engagement Survey

- SDM ACP Self-Efficacy Survey

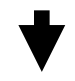

Randomise: Plan Well Guide decision support intervention versus usual care

Intervention:

- Immediate Plan Well

tool intervention is

administered by a

trained clinical research

assistant

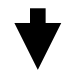

\section{Usual care:}

- Delayed intervention

$\mathbf{7}$

Brief reminder call 1-2 Brief reminder call 1-2 weeks weeks prior to appointment prior to appointment

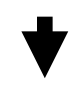

Postintervention follow-up Postrandomisation follow-up (12 Weeks):

(12 Weeks):

- Follow-up Patient ACP Engagement Survey

- Follow-up SDM ACP Engagement Survey

- Follow-up SDM ACP Self-Efficacy Survey
- Follow-up Patient ACP Engagement Survey

- Follow-up SDM ACP Engagement Survey

- Follow-up SDM ACP SelfEfficacy Survey

- Offered Plan Well Guide decision support intervention on ACP created by the provincial Hospice Palliative Care Association, which describes the provincial legal context of ACP.
Blinded outcome ascertainment is conducted at week 12 for both groups, by a trained research assistant. The Plan Well decision support intervention visit will be conducted in the clinic from which the patient was recruited at a mutually convenient time.

\section{Study settings}

Recruitment will occur in outpatient settings in one area (Hamilton, Burlington) in Southern Ontario, Canada including family practice, cardiology outpatient care and cancer pain and symptom management. The city of Hamilton (population 500 000) has two multisite general tertiary hospitals. There are approximately 400 family physicians in the city. Most people in Canada and Ontario have a regular primary care provider and a primary care referral is required to access specialist consultation. ${ }^{34}$ In all of these settings, there are patients presenting with chronic conditions that would be expected to shorten life expectancy and at some point require decision-making, and who could, therefore, benefit from engaging in ACP.

\section{Eligibility criteria}

Participants are patients aged 65 years and older attending ambulatory healthcare settings (family physicians, cancer clinic, cardiology clinic and respirology clinic) who could benefit from ACP as judged by their clinician and the patient's identified SDM. In order to compare results to other studies of ACP tools using similar methods and measures, eligible patients will be 65 years of age and older. $^{35}$ To be eligible the SDM must be 18 years or age or older. In addition to the age criterion and in keeping with recommendations for when clinicians should consider ACP conversations, ${ }^{36} 37$ clinicians are asked to identify patients they consider 'high risk' for probability of a decline in health that may require hospitalisation. Each physician or clinic will establish their high-risk criteria based on age, comorbidities, change in health status or recent hospitalisations.

Additional eligibility criteria include being cognitively able to consent to research (as perceived by the referring clinician's judgement), able to communicate in English, and having adequate hearing and vision (with aids) to be able to interact with the research staff. Exclusion criteria include cognitive impairment that precludes giving informed consent and inability to communicate in English. When the research assistants contact patients to explain the study in full and obtain informed consent, they will also ensure that the patient is suitable from a cognitive and language perspective, using their judgement of the patient's understanding of the study explanation. Efforts will be made to include patients with hearing or visual impairments by arranging for data collection to occur in person rather than on the telephone if the patient requires.

\section{Recruitment}

Patients will be recruited prospectively by the clinicians involved in their care, when the patient attends a study 
site for an outpatient visit. Privacy laws impede the ability of researchers to obtain patient names from healthcare settings without the patient's prior consent, therefore, creation of a sampling frame from clinic records to contact patients is not possible, and it is not feasible for the clinicians in the study sites to contact lists of patients. Clinicians will be provided with the scripted language to introduce the study during clinic encounters and ask potentially eligible patients if they would be willing to be contacted by the researchers to learn more. Posters about the study will be provided to clinics for advertising. At regular intervals determined with each clinic (approximately weekly), names and contact information of patients who have agreed to be contacted will be forwarded to the research team.

Research assistants will telephone patients who have agreed to be contacted. At this point, the research assistant will confirm eligibility, explain the study using the research ethics board approved information sheet, and obtain initial verbal consent to participate. The patient will then be asked to approach the person they believe would be their SDM and ask this person for permission to be contacted by the research assistant. The research assistant will then contact the prospective SDM by telephone to confirm eligibility, explain the study and obtain verbal consent. Consent refusals, and reason, will be recorded in a screening log.

\section{Randomisation procedures}

After consent has been obtained and baseline measures have been administered to both the patient and SDM, the participating 'dyad' will be randomised. Randomisation will be concealed and blocked using variable block size.

\section{Blinding}

Study participants will not be blinded to their group allocation. To ensure blinding of the outcome assessment, the research assistant, who conducts follow-up interviews, will not be the same person who completed the baseline interview or randomisation for that participant. Investigators will be blinded to group allocation of the participants.

\section{Study intervention}

The main component of the intervention is the guided use of the Plan Well Guide. A trained research nurse will run the interactive decision aid for the patient and SDM on a laptop computer and complete the structured paper-based values clarification and preference questions with the patient when prompted by the decision aid. The output of the decision aid is a structured 'Dear Doctor' letter, which is given to the patient for their own use. The research nurse who facilitates the use of the Plan Well Guide may assist the patient through their deliberations about the values and preferences questions and review the information from sections of the tool as needed until the patient is comfortable expressing a preference but will not provide advice about person medical decisions. If questions of this nature arise, the research nurse will remind the patient of the 'Dear Doctor' letter to be used as a discussion aid with the patient's physician. The patient and SDM are informed verbally and in writing that this summary letter does not represent consent to treatment and has no legal standing and that the intent is to inform possible future decision-making.

The Plan Well Guide was designed with input from 24 older adult patients in primary care practices who had serious health conditions. In addition, 42 healthcare professionals with expertise in critical care medicine, nursing, geriatrics, family medicine and palliative care participated in the focus groups, webinars, and interviews and provided input on the decision aid.

The Plan Well Guide (1) describes why preparation for decision-making is important, the difference between serious illness and terminal care, and the significance of making decision under conditions of uncertainty, (2) defines personal values, why these are important in decision-making and how trade-offs are sometimes required, (3) provides education on typical end-of-life trajectories and discusses conditions of uncertainty under which healthcare decisions are often made (4) provides education about different approaches to care in hospital (resuscitative/intensive care, medical care and comfort care) and (5) guides the patient through a values and preference elicitation process, explicitly linking values to preferences using rating scales.

The tool describes treatment options (resuscitative/ intensive care, medical care and comfort care) in detail with information about the nature, location, harms, benefits and associated outcomes of the different options, using verbal information, text and visual images (eg, photographs of hospital rooms). The information included about outcomes of the different treatment options is supported by the medical literature and expressed in probabilistic terms and presented visually.

Since the treatment options are preference sensitive and preferences should be aligned with values, a short value clarification tool is used that includes an explanation of values and their relationship to treatment decisions, and examples of different end-of-life values (adapted from Scheunemann et $a l^{38}$ ). The presentation explains how certain values compete or conflict with each other, and patients are asked to rate on seven-point Likert-type scales (1) the degree to which quality of life is more or less important to them compared with quantity of life and (2) whether a natural death versus a machine-supported death is more important. To aid in future clinical decision-making and to make the linkage between values and preferences more explicit, a system of grids is used by the research nurse to map the ratings of importance on the values questions to the treatment approach (intensive, medical and comfort) which would most align with the values. The grids are used to provide structured guidance to the patient helping them link their stated values to reasonable treatment preferences. If the patient indicates a preference for CPR, a CPR information video is shown to provide detailed information about the benefits and 
risks of this procedure.$^{39}$ If the patient feels there is discordance in the treatment preference highlighted on the grids (eg, the first grid suggested the preferred medical option was comfort care and the second grid indicated medical care), the research nurse reviews the patient's values, and which values are most important to determine if they would like any changes. The final step is to elicit a treatment preference for the use of life-sustaining treatments. A taxonomy describing different levels of the use or non-use of life-sustaining treatments is used, which was developed with input from medical experts and has been used extensively in our prior research. ${ }^{143140}$

\section{Usual care}

If the patient is randomised to usual care, an appointment for the follow-up administration of outcome measures to the patient and SDM is made for 3 months later. At completion of the 3-month follow-up, usual care group participants will be offered the Plan Well Guide decision support intervention.

\section{Intervention fidelity and data collection methods}

All staff members are trained on the study protocol and measures, and perform at least one mock intervention session, including data collection, with the senior project manager or another experienced senior staff member in the investigators' research programme. Research data are captured on paper and entered into a web-based software program called Research Electronic Data Capture (REDCap), managed by the Clinical Evaluation Research Unit (DKH director) at Kingston General Hospital, Kingston, Ontario, Canada. All patients will be given a unique, non-identifying patient identification number that will be removed from any personally identifying information. Personal identifying information will be stored in a Microsoft Access database stored behind a firewall at McMaster University (Department of Family Medicine server). REDCap will be programmed so that fields cannot be skipped on data entry. A $10 \%$ random sample of data will be checked for data entry accuracy.

All data collection will be done through participant interviews primarily by telephone, or in-person if needed to accommodate participant's accessibility needs.

The staff team has experience with interview data collection using the instruments from other studies. They will meet regularly to discuss and resolve issues relating to quality of data collection.

\section{Outcomes and measures}

The primary outcome will be evaluated in the SDM. We will use a validated 'SDM ACP Engagement Survey' measure of behavioural change that conveys SDM engagement (knowledge, contemplation, self-efficacy and readiness) for their role with respect to the patient. ${ }^{32}$ The survey is based on a previously validated patient ACP Engagement Survey, which measures the same ACP behavioural change as well as several ACP actions, including identifying the SMD, identifying values and goals for medical care, and documenting one's wishes. Validity, reliability and responsiveness of the patient ACP Engagement Survey and validation of short versions have been previously described..$^{35} 4142$ The patient version was adapted to a version for SDMs by modifying the language of items to reflect the SDM's perspective, and reducing items and confirming factors through psychometric analyses using data from 65 respondents who were family members of patients in a hospital inpatient setting. ${ }^{32}$ The version contains 17 items, with three factors that explained $91 \%$ of variance.

Key secondary outcomes include change in ACP engagement of the patient, using a 15-item version of the patient ACP Engagement Survey. Details of the methods and properties have been published. ${ }^{43}$ This shorter version was created from a longer original version by systematically eliminating questions based on face validity, item non-response, redundancy, ceiling effects and factor analysis. The 15-item version demonstrated responsiveness to change, Cronbach's alpha was 0.92 and correlation of the mean score to the original version was 0.91 .

We will use an adapted version of the SURE-Decisional Conflict Scale ${ }^{44}$ with modified wording appropriate for the SDM and on a five-point scale (not at all confident to very confident). This survey augments the primary outcome measure of SDM ACP engagement and provides an opportunity for further validation (ie, concurrent) of the primary outcome measure survey.

Other secondary outcomes to be measured after the intervention, only in those who receive it include global rating of satisfaction with the Plan Well Guide decision support intervention and endorsement of the tool, using Likert-type scales. At the end of the Plan Well decision support intervention, we will also evaluate decisional conflict in the context of life-sustaining treatment using the SURE-Decisional Conflict Scale. ${ }^{44}$

To describe the sample, baseline information collected will include age, sex, living location, education level, health literacy, language spoken and single item global ratings of quality of life and self-reported health.

All study measures and timing of administration in the patient and SDM are listed in table 2.

\section{Follow-up}

At 8 weeks following the baseline, a research assistant will contact the patients and SDMs in both groups to set up a time to administer the outcome assessment via telephone in 4 weeks. We chose 12 weeks for follow-up to allow time for reflection by the patient and SDM on the intervention and additional communication between them that may enhance the SDM's perception of confidence and readiness in the SDM role.

Five attempts over 2 weeks at different times of day will be made to reach the patient and SDM. If the participants cannot be reached after these attempts, they will be considered lost to follow-up. If one of the dyad completes follow-up, their data will be used, as appropriate for analyses of primary or secondary outcomes. 
Table 2 Study measures and timing of administration

\begin{tabular}{|c|c|c|c|c|}
\hline Measure & $\begin{array}{l}\text { Baseline- } \\
\text { patient }\end{array}$ & Baseline- SDM & $\begin{array}{l}\text { Follow-up- } \\
\text { Patient }\end{array}$ & Follow-up- SDM \\
\hline $\begin{array}{l}\text { Demographic information: } \\
\text { Age (years), sex, marital status, highest completed education, } \\
\text { type of home, language spoken, health literacy }\end{array}$ & $\mathrm{X}$ & $\mathrm{X}$ & - & - \\
\hline Self-reported health and quality of life & $\mathrm{X}$ & & & \\
\hline $\begin{array}{l}\text { Primary outcome: } \\
\text { SDM ACP engagement } \\
\text { (Modified from the ACP Engagement Survey for SDM, }{ }^{43} \\
\text { eg, knowledge of patient's wishes, and confidence making } \\
\text { decisions, 5-point Likert scales) }\end{array}$ & - & $\mathrm{X}$ & - & $\mathrm{X}$ \\
\hline $\begin{array}{l}\text { Secondary outcome: } \\
\text { SDM Decisional Conflict (Modified Decisional Conflict Scale }{ }^{49} \text { in } \\
\text { SDM) }\end{array}$ & - & $\mathrm{X}$ & - & $\mathrm{X}$ \\
\hline $\begin{array}{l}\text { Secondary outcome: } \\
\text { Decisional Conflict (Modified Decisional Conflict Scale }{ }^{44} \text { ) }\end{array}$ & - & - & $\mathrm{X}$ & - \\
\hline $\begin{array}{l}\text { Implementation outcome: Satisfaction and endorsement of } \\
\text { intervention }\end{array}$ & - & - & $\mathrm{X}$ & - \\
\hline
\end{tabular}

ACP, advance care planning; SDM, substitute decision-maker.

\section{Sample size}

The sample size is based on the mean difference between groups on the change in score for the SDM ACP Engagement Survey. Based on our previous non-randomised study of patients using paper and web-based ACP tools with the primary outcome of the patient ACP Engagement Survey with effects sizes (Cohen's d) of 0.6 and greater, the study will be powered to detect a moderate to large effect size. The sample size needed for a normally distributed outcome, to detect a moderate effect size (0.6 in the following formula) with $80 \%$ power at a two-sided alpha $=0.05$ is $16 /\left(0.6^{2}\right)$ is 45 per group (or $38 /$ group if 0.65 effect size). (http://rpsychologist.com/d3/ cohend/) To allow for $20 \%$ lost to follow-up, we aim to enrol 54 patients per group.

\section{Statistical analysis}

Results from this trial will be reported in accordance with the Consolidated Standards of Reporting Trials statements. ${ }^{45}$ Baseline characteristics of the study participants will be reported in terms of mean (SD) or median (first quartile and third quartile), depending on the distribution, for continuous variables and count (percentage) for categorical variables.

The primary analyses will be performed using intention-to-treat approach. Analyses will be performed on an intention-to-treat basis. No interim analyses are planned. Multiple imputation method will be used to impute the missing data assuming missing data follow a missing at random pattern. In total, five datasets will be generated, and the pooled estimate will be reported.
The primary and secondary continuous outcomes will be analysed using linear regression. The mean difference along with $95 \%$ CI will be reported. All statistical tests will be two sided at the level of significance 0.05 . All $\mathrm{p}$ values will be reported to three decimal places with less than 0.001 reported as $<0.0010$. $^{46}$

Several sensitivity analyses will be performed to assess the robustness of the results of primary analyses. The primary and secondary outcomes will be analysed using per-protocol approach. We will analyse results with and without the participant dyads who serve as each other's SDM. We will use analysis of covariance, to adjust for study centre. Finally, we will analyse the outcomes by imputing missing data using last observation carried forward method.

\section{PATIENT AND PUBLIC INVOLVEMENT}

Neither patient populations nor the public were involved in the development of this research protocol.

\section{ETHICS AND DISSEMINATION}

Written informed consent will be obtained for all participants. Protocol modifications will be handled by amendments to the ethics board.

The investigators on this trial include physicians in family medicine, palliative care and critical care, health services researchers and a biostatistician. Given the low risk nature of the intervention, there is no formal data safety and monitoring board.

Manuscripts will be published in peer-reviewed journals, one reporting results for the primary outcome of 
SDM ACP engagement and another for the secondary outcomes. The findings will also be publicised through our network of stakeholders at www.thecarenet.ca

\section{DISCUSSION}

One of the most important aspects of preparing for future healthcare decisions is the education and engagement of SDMs, so that they understand the authentic values and informed preferences of their loved one. If this phase II randomised trial demonstrates that the decision aid improves SDM engagement in ACP through increased confidence and readiness to be involved in decision-making if needed in the future for their loved one, this tool should be evaluated to determine its effectiveness at improving SDM that leads to care consistent with a patient's values and wishes.

Our trial has several strengths. This trial is novel in that it specifically targets the SDMs of patients who are likely to benefit from ACP and uses a measure of SDM engagement in ACP in relation to the patient for whom they may need to make medical decisions in the future. Participants are being recruited from a variety of outpatient settings in a pragmatic way that mirrors how ACP might be undertaken in these clinical settings. We are using a delayed intervention process as a control to allow all participants to eventually receive the intervention if they wish. The trial also has potential limitations. It is not possible to blind participants to group allocation. The Plan Well Guide is facilitated by a research nurse and the effects may differ if it is self-administered. The tool is scripted and structured with the intention of non-facilitated use; however, the interactive online version was not available when this trial began. The decision aid intervention has not been translated to languages other than English or adapted for varying cultures, therefore, study participants may represent the typical Caucasian English speaking more educated population as found in many previous studies of ACP. ${ }^{47}$ Successful models of adapting ACP tools for other populations exist ${ }^{48}$ and should be considered in the future.

\section{Trial status}

Recruitment and delivery of the intervention are ongoing. We started recruitment of patients and SDM in February 2018 and expect to complete recruitment of the last participant by October 2019. At the time of this manuscript submission, 53 patient/SDM dyads had been randomised.

\author{
Author affiliations \\ ${ }^{1}$ Family Medicine, McMaster University, Hamilton, Ontario, Canada \\ ${ }^{2}$ Division of Palliative Care, McMaster University, Hamilton, Ontario, Canada \\ ${ }^{3}$ Family and Community Medicine, University of Toronto, Toronto, Ontario, Canada \\ ${ }^{4}$ Department of Family Medicine, University of Calgary Cumming School of \\ Medicine, Calgary, Alberta, Canada \\ ${ }^{5}$ Critical Care, Queen's University, Kingston, Ontario, Canada \\ ${ }^{6}$ Clinical Evaluation Research Unit, Kingston General Hospital, Kingston, Ontario, \\ Canada
}

Contributors $\mathrm{MH}$ and DKH conceived of the study and wrote the trial protocol paper. MH, MS, CB, SB, DE, NA and AT contributed to the refinement of the study protocol and will contribute to interpretation of results and dissemination. SB will conduct statistical analyses. MS, CB, SB, DE, NA, AT and DKH contributed to critically revising the protocol manuscript and approved the final version.

Funding This work was supported by the Canadian Institutes of Health Research grant number PHE-135930.

Disclaimer The funder had no role in the design of the study. The funder will have no role in the collection, management, analysis or interpretation of data, writing of any reports or the decision to submit any report for publication.

Competing interests None declared.

Patient consent for publication Not required.

Ethics approval This study has been approved by the Hamilton Integrated Research Ethics Board (number 2017-3714-GRA).

Provenance and peer review Not commissioned; externally peer reviewed.

Open access This is an open access article distributed in accordance with the Creative Commons Attribution Non Commercial (CC BY-NC 4.0) license, which permits others to distribute, remix, adapt, build upon this work non-commercially, and license their derivative works on different terms, provided the original work is properly cited, appropriate credit is given, any changes made indicated, and the use is non-commercial. See: http://creativecommons.org/licenses/by-nc/4.0/.

\section{REFERENCES}

1. Wenger NS, Oye RK, Bellamy PE, et al. Prior capacity of patients lacking decision making ability early in hospitalization: implications for advance Directive administration. The support Investigators. study to understand prognoses and preferences for outcomes and risks of treatments. J Gen Intern Med 1994;9:539-43.

2. Raymont V, Bingley W, Buchanan A, et al. Prevalence of mental incapacity in medical inpatients and associated risk factors: crosssectional study. The Lancet 2004;364:1421-7.

3. Fried TR, Redding CA, Robbins ML, et al. Agreement between older persons and their surrogate decision-makers regarding participation in advance care planning. J Am Geriatr Soc 2011;59:1105-9.

4. Shalowitz DI, Garrett-Mayer E, Wendler D, et al. The accuracy of surrogate decision makers. Arch Intern Med 2006;166:493.

5. Fried TR, Zenoni M, lannone L, et al. Engagement in Advance Care Planning and Surrogates' Knowledge of Patients' Treatment Goals. J Am Geriatr Soc 2017;65:1712-8.

6. Sudore RL, Lum HD, You JJ, et al. Defining advance care planning for adults: a consensus definition from a multidisciplinary Delphi panel. J Pain Symptom Manage 2017;53:821-32.

7. Hammes BJ, Rooney BL. Death and end-of-life planning in one midwestern community. Arch Intern Med 1998;158:383-90.

8. Silveira MJ, Kim SYH, Langa KM. Advance directives and outcomes of surrogate decision making before death. N Engl J Med 2010;362:1211-8.

9. Detering KM, Hancock AD, Reade MC, et al. The impact of advance care planning on end of life care in elderly patients: randomised controlled trial. BMJ 2010;340:c1345.

10. Metzger M, Song M-K, Ward S, et al. A randomized controlled pilot trial to improve advance care planning for LVAD patients and their surrogates. Heart Lung 2016;45:186-92.

11. Teno JM, Fisher ES, Hamel MB, et al. Medical care inconsistent with patients' treatment goals: association with 1-year Medicare resource use and survival. J Am Geriatr Soc 2002;50:496-500.

12. Houben CHM, Spruit MA, Groenen MTJ, et al. Efficacy of advance care planning: a systematic review and meta-analysis. J Am Med Dir Assoc 2014;15:477-89.

13. You JJ, Dodek P, Lamontagne F, et al. What really matters in endof-life discussions? perspectives of patients in hospital with serious illness and their families. Can Med Assoc J 2014;186:E679-87.

14. Heyland DK, Dodek P, Mehta S, et al. Admission of the very elderly to the intensive care unit: family members' perspectives on clinical decision-making from a multicenter cohort study. Palliat Med 2015;29:324-35.

15. Kobewka DM, van Walraven C, Turnbull J, et al. Quality gaps identified through mortality review. BMJ Qual Saf 2017;26:141-9.

16. Sharma RK, Freedman VA, Mor V, et al. Association of racial differences with end-of-life care quality in the United States. JAMA Intern Med 2017;177:1858.

17. Khandelwal N, Curtis JR, Freedman VA, et al. How often is end-of-life care in the United States inconsistent with patients' goals of care? J Palliat Med 2017;20:1400-4. 
18. Heyland DK, Heyland R, Dodek P, et al. Discordance between patients' stated values and treatment preferences for end-of-life care: results of a multicentre survey. BMJ Support Palliat Care 2017:7:292-9.

19. Howard M, Bansback N, Tan A, et al. Recognizing difficult trade-offs: values and treatment preferences for end-of-life care in a multi-site survey of adult patients in family practices. BMC Med Inform Decis Mak 2017;17:164.

20. Jones GK, Brewer KL, Garrison HG. Public expectations of survival following cardiopulmonary resuscitation. Acad Emergency Med 2000;7:48-53.

21. Roulston E. Canadians' views on palliative care. J Palliat Med 2018;21:S-9-0.

22. Perkins HS. Controlling death: the false promise of advance directives. Ann Intern Med 2007:147:51-7.

23. Winter L, Parks SM, Diamond JJ. Ask a different question, get a different answer: why living wills are poor guides to care preferences at the end of life. J Palliat Med 2010;13:567-72.

24. Hickman RL, Daly BJ, Lee E. Decisional conflict and regret: consequences of surrogate decision making for the chronically critically ill. Appl Nurs Res 2012;25:271-5.

25. Stacey D, Légaré F, Col NF, et al. Decision AIDS for people facing health treatment or screening decisions. Cochrane database of systematic reviews 2014;1.

26. Cardona-Morrell M, Benfatti-Olivato G, Jansen J, et al. A systematic review of effectiveness of decision AIDS to assist older patients at the end of life. Patient Educ Couns 2017;100:425-35.

27. Wendler D, Rid A. Systematic review: the effect on surrogates of making treatment decisions for others. Ann Intern Med 2011;154:336-46.

28. Lewis $\mathrm{E}$, Cardona-Morrell $\mathrm{M}$, Ong KY, et al. Evidence still insufficient that advance care documentation leads to engagement of healthcare professionals in end-of-life discussions: a systematic review. Palliat Med 2016;30:807-24.

29. McMahan RD, Knight SJ, Fried TR, et al. Advance care planning beyond advance directives: perspectives from patients and surrogates. J Pain Symptom Manage 2013;46:355-65.

30. Kelly B, Rid A, Wendler D. Systematic review: individuals' goals for surrogate decision-making. J Am Geriatr Soc 2012;60:884-95.

31. Sudore RL, Fried TR. Redefining the "planning" in advance care planning: preparing for end-of-life decision making. Ann Intern Med 2010;153:256.

32. Van Scoy LJ, Day AG, Howard M, et al. The development and validation of the advance care planning engagement survey for surrogate decision makers. J Pain Symptom Manage 2018.

33. Chan A-W, Tetzlaff JM, Gøtzsche PC, et al. Spirit 2013 explanation and elaboration: guidance for protocols of clinical trials. $B M J$ 2013;346:e7586.
34. Hutchison B, Levesque J-F, Strumpf E, et al. Primary health care in Canada: systems in motion. Milbank Q 2011;89:256-88.

35. Sudore RL, Boscardin J, Feuz MA, et al. Effect of the prepare website vs an Easy-to-Read advance Directive on advance care planning documentation and engagement among veterans: a randomized clinical trial. JAMA Intern Med 2017;177:1102-9.

36. Downar J, Goldman R, Pinto R, et al. The "surprise question" for predicting death in seriously ill patients: a systematic review and meta-analysis. Can Med Assoc J 2017;189:E484-93.

37. Bernacki RE, Block SD. Communication about serious illness care goals: a review and synthesis of best practices. JAMA Intern Med 2014;174:1994-2003.

38. Scheunemann LP, Arnold RM, White DB. The facilitated values history. Am J Respir Crit Care Med 2012;186:480-6.

39. You JJ, Swinton M, Mantle C, et al. P043 effect of a video decision aid on clinical decision-making about cardiopulmonary resuscitation. $J$ Pain Symptom Manage 2016;52:e77.

40. Heyland DK, Barwich D, Pichora D, et al. Failure to engage hospitalized elderly patients and their families in advance care planning. JAMA Intern Med 2013;173:778.

41. Sudore RL, Knight SJ, McMahan RD, et al. A novel website to prepare diverse older adults for decision making and advance care planning: a pilot study. J Pain Symptom Manage 2014:47:674-86.

42. Sudore RL, Stewart AL, Knight SJ, et al. Development and validation of a questionnaire to detect behavior change in multiple advance care planning behaviors. PLoS One 2013;8:e72465.

43. Sudore RL, Heyland DK, Barnes DE, et al. Measuring advance care planning: optimizing the advance care planning engagement survey. $J$ Pain Symptom Manage 2017;53:669-81.

44. Légaré $\mathrm{F}$, Kearing $\mathrm{S}$, Clay $\mathrm{K}$, et al. Are you sure?: assessing patient decisional conflict with a 4-item screening test. Can Fam Physician 2010;56:e308-14.

45. Moher D, Hopewell S, Schulz KF, et al. Consort 2010 explanation and elaboration: updated guidelines for reporting parallel group randomised trials. BMJ 2010;340:c869.

46. R Foundation for Statistical Computing. A language and environment for statistical computing. Vienna, Austria: R Core Team, 2018.

47. Lin C-P, Evans CJ, Koffman J, et al. The conceptual models and mechanisms of action that underpin advance care planning for cancer patients: a systematic review of randomised controlled trials. Palliat Med 2019;33:5-23

48. Sudore RL, Schillinger D, Katen MT, et al. Engaging diverse English- and Spanish-speaking older adults in advance care planning: the prepare randomized clinical trial. JAMA Intern Med 2018;178:1616-25.

49. O'Connor A. User manual - decision self-efficacy scale. Ottawa, 1995. Available: https://decisionaid.ohri.ca/docs/develop/User Manuals/UM_Decision_SelfEfficacy.pdf [Accessed 8 May 2019]. 